\title{
The Origin of the barrier of rotation of alpha carbonyl methyl groups
}

\section{Bruno A. Piscelli, Rodrigo A. Cormanich}

\section{Abstract}

The knowledge about the effects that rule internal rotation of methyl groups is the key in understanding physical chemistry properties and reactivity of molecules. Using this as motivation, different substituted acetyl groups were studied with NBO theory and the effects that govern conformational preferences calculated by computational methods.

\section{Key words:}

NBO, Computational Chemistry, Physical Organic Chemistry.

\section{Introduction}

The understanding of the effects that govern the internal rotation of methyl groups is one of the foundations of conformational analysis and has been an aim of study for several decades and the debate in the literature remain a matter of dispute even for the simplest organic molecules. ${ }^{1}$ Although one can find some experimental and theoretical studies in the literature ${ }^{2}$ less attention has been given to the origins of the internal rotation of methyl groups in alpha position to $\mathrm{C}=\mathrm{O}$ bonds and to the correlation of such rotations for different carbonyl functional groups.

The methyl group can adopt two geometries in relationship to the carbonyl moiety, the eclipsed and the bisected, as shown in the Figure 1. It is well known that the eclipsed is the most stable one, even when large groups are attached to the alpha $\mathrm{C}$ atom, and different explanations raised in the literature to explain such an unexpected behaviour. In this way, the present study aims to elucidate the effects that rule the preference for eclipsed conformations by using high level theoretical calculations.

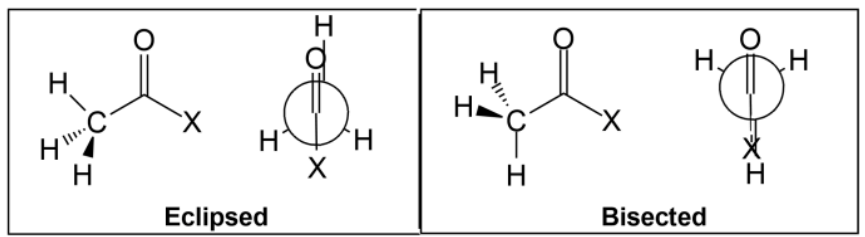

Fig. 1: Geometrical representations of the eclipsed and bisected conformers for methyl groups $\alpha$ to carbonyls. The $X$ groups proposed for study are $\mathrm{H}, \mathrm{F}, \mathrm{Cl}, \mathrm{Br}, \mathrm{CH}_{3}, \mathrm{CF}_{3}$, $\mathrm{CH}_{2} \mathrm{CF}_{3}, \mathrm{NH}_{2}, \mathrm{NHCH}_{3}, \mathrm{~N}\left(\mathrm{CH}_{3}\right)_{2}, \mathrm{OH}, \mathrm{OF}, \mathrm{OCH}_{3}, \mathrm{OCF}_{3}$, $\mathrm{OCH}_{2} \mathrm{CH}_{3}$ and $\mathrm{OCH}_{2} \mathrm{CF}_{3}$

\section{Results and Discussion}

Molecules that have s-cis and s-trans conformers, as shown in Figure 2, had both conformers energies calculated separately.

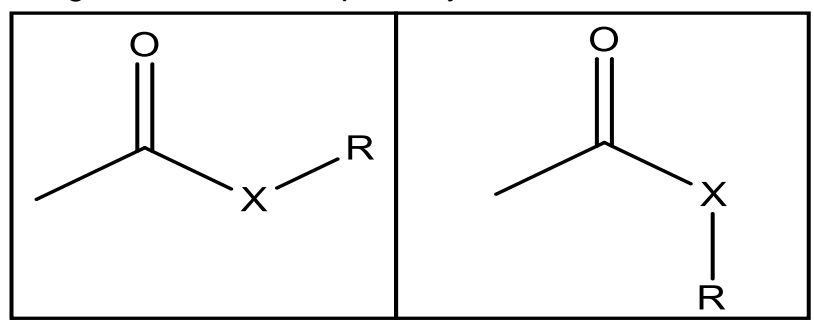

Fig. 2: Geometrical representations of the s-cis (left) and $\mathrm{s}$-trans (right) conformers for the $\mathrm{X}$ substituents.
The geometries of all molecules were optimized at the M06-2X/aug-cc-pVTZ level and the energies calculated according to the NBO theory, using the commercial software Gaussian 09.

The total energy (TE), hyperconjugation energy (NL), electrostatic energy (NCE) and steric energy for 4 molecules studied are shown as an example in Table 1.

Table 1. Relative $\mathrm{NBO}$ energies calculated for $\mathrm{AcCl}$, $\mathrm{AcCF}_{3}, \mathrm{AcNH}_{2}$ and $\mathrm{AcOH}$ (in kcal mol${ }^{-1}$ ).

\begin{tabular}{cccccc} 
Molecule & Conformer & TE & NL & NCE & Steric \\
\multirow{2}{*}{ AcCl } & Eclipsed & 0.00 & 3.03 & 0.46 & 0.00 \\
& Bisected & 0.19 & 0.00 & 0.00 & 0.13 \\
AcCF $_{3}$ & Eclipsed & 0.00 & 1.03 & 0.15 & 1.18 \\
& Bisected & 0.77 & 0.00 & 0.00 & 0.00 \\
AcNH $_{2}$ & Eclipsed & 0.00 & 0.00 & 0.00 & 0.25 \\
& Bisected & 0.02 & 0.85 & 3.33 & 0.00 \\
AcOH & Eclipsed & 0.00 & 1.89 & 0.50 & 0.00 \\
(cis) & Bisected & 0.47 & 1.18 & 0.00 & 0.34 \\
AcOH & Eclipsed & 5.18 & 0.92 & 18.20 & 1.37 \\
(trans) & Bisected & 5.94 & 0.00 & 19.32 & 1.14 \\
\hline
\end{tabular}

As shown in the Table 1, the major effect that governs the conformational preference is hyperconjugation $(\mathrm{NL})$, in particular the $\sigma_{\mathrm{CH} \rightarrow \sigma^{*} \mathrm{CX}}$, $\sigma_{\mathrm{CX}} \rightarrow \sigma^{*}{ }_{\mathrm{CH}}, \sigma_{\mathrm{CH} \rightarrow} \rightarrow \Pi^{*} \mathrm{CO}$ and $\Pi \mathrm{CO} \rightarrow \sigma^{*} \mathrm{CH}$ interactions, since the overlap between these orbitals is poor in the bisected conformers and, consequently, these hyperconjugative interactions are more stabilizing for eclipsed conformers.

\section{Conclusions}

For the compounds studied in this study, the eclipsed conformer is always more stable than the bisected. Hyperconjugation play the most important role in this preference. In addition, electrostatic effects are of secondary importance, while steric effects are negligible.

\section{Acknowledgements}

The authors acknowledge FAPESP and FAEPEX for the financial support and SDumont, CESUP and CENAPAD-SP for the computational facilities. DOI:10.1021/ja00501a009.

2 G. J. Karabatsos and N. Hsi, J. Am. Chem. Soc., $1965,87,2864-2870$. 